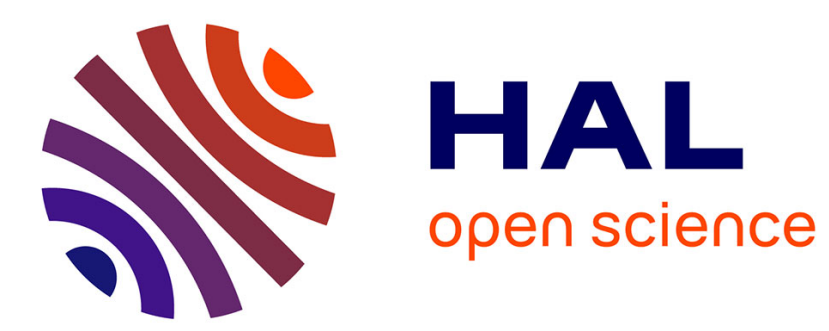

\title{
Introduction to the theory of random particle methods for Boltzmann equation
}

Benoît Perthame

\section{To cite this version:}

Benoit Perthame. Introduction to the theory of random particle methods for Boltzmann equation.

[Research Report] RR-2218, INRIA. 1994. inria-00074452

\section{HAL Id: inria-00074452 \\ https://hal.inria.fr/inria-00074452}

Submitted on 24 May 2006

HAL is a multi-disciplinary open access archive for the deposit and dissemination of scientific research documents, whether they are published or not. The documents may come from teaching and research institutions in France or abroad, or from public or private research centers.
L'archive ouverte pluridisciplinaire HAL, est destinée au dépôt et à la diffusion de documents scientifiques de niveau recherche, publiés ou non, émanant des établissements d'enseignement et de recherche français ou étrangers, des laboratoires publics ou privés. 


\section{Introduction to the Theory of Random Particle Methods for Boltzmann Equation}

Benoît PERTHAME

$\mathbf{N}^{\circ} 2218$

Mars 1994

PROGRAMME 6

Calcul scientifique, modélisation et

logiciels numériques

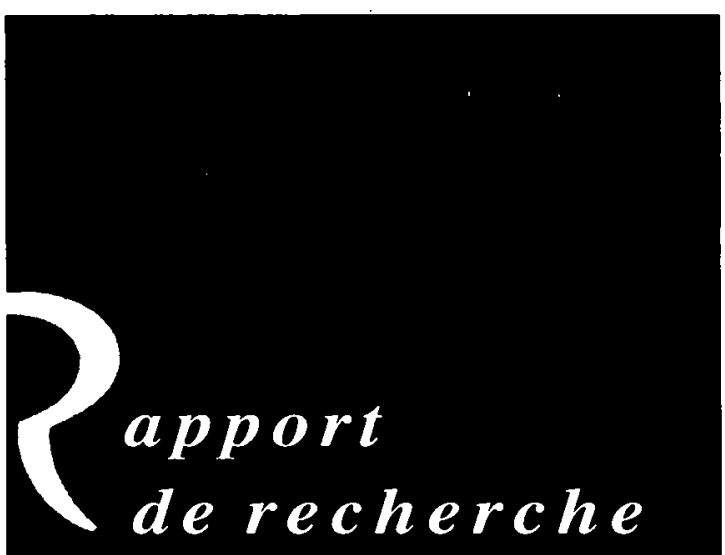




\title{
Introduction to the Theory of Random Particle Methods for Boltzmann Equation
}

\author{
Benoît Perthame \\ Université Pierre et Marie Curie \\ Laboratoire d'Analyse Numérique \\ CNRS UA189, T.55/65, 5e étage \\ 4 , place Jussieu \\ F75252 PARIS Cedex 05 \\ and \\ INRIA, Projet MENUSIN \\ B.P. 105 \\ F78153 LE CHESNAY Cedex
}

\section{Abstract :}

We present theoretical derivations of several random particle methods for solving the Boltzmann equation, including Direct Simulation Monte Carlo methods and a method derived directly from Boltzmann equation. The DSMC methods are presented as discretizations of the master equations.

\section{Introduction à la Théorie des Méthodes Particulaires Aléatoires pour les Equations de Boltzmann}

\section{Résumé :}

Nous présentons des dérivations théoriques de plusieurs méthodes particulaires aléatoires pour la résolution des équations de Boltzmann, y compris les méthodes DSMC de simulation directe par Monte-Carlo et une méthode directement dérivée des équations de Boltzmann. Les méthodes DSMC sont introduites comme discrétisations des équations Master. 


\section{Introduction}

The object of this text is to present how several classical resolution methods for the Boltzmann equation can be derived theoretically. We focuss on the methods based on a particle approximation of the density which means as a sum of Dirac masses, and where the integrations resulting from Boltzmann kernel are numerically performed using a random method.

We will derive these methods either from the master equations or from the Boltzmann equation directly.

Due to the large number of variables in the Boltzmann equation ( $t$ for time, two three-dimensional vectors $x$ for space anv $v$ for velocity, also a onedimensional internal energy parameter is needed for polyatomic gases), much of the methods are based on a Monte Carlo procedure, or at least random integrations, at several levels. This is the case of those methods developed departing from the master equations and called Direct Simulation Monte Carlo methods (DSMC in short), see Bird [5], Koura [15], Nanbu [17], Ivanov and Rogasinsky [14]. Since the master equations are linear, like in neutron transport, the solution can be represented with the help of a jump markov process and the DSMC methods mimic this process. After Nanbu's method [16], the tendancy has been to relate more closely to Boltzmann equations (see Babovsky [2], Babovsky and Mlner [3], Neunzert et al. [18]) but random methods are still used.

These methods relying on particle approximations have the general advantage to put discretization points where they are needed (see also the other papers in this issue). For instance, for hypersonic flows in a rarefied atmosphere, the average flow velocity away from the obstacle can be around a Mach number of 25, but is zero close to the obstacle (see figure 1). This problem would require a huge number of grid points in $v$ for a finite difference method with a fixed grid. On the other hand, particle methods always provide fluctuating results. This is due of course to the Monte Carlo, procedure used to solve the collision operator, but also because the transport operator is solved exactly. Hence no numerical diffusion is present to smooth out the results. Compare for instance finite volume schemes for the Euler limit of the Boltzmann equation with particle-based schemes (Pullin [22], Coron and Perthame [8], Perthame [20] : so many particles are needed that particle emthods are not competitive in the fluid regime.

In order to avoid fluctuations, several authors proposed different approaches based on finite volumes, finite elements or finite differences, especially to compute transitory regimes or problems where fluctuations are costly to avoir (low mach numbers for instance, or small variations of the flow velocity). Let us give some very incomplete set of references for such methods. Aristov and Tcheremissine [1] have developed an important effort in computing Boltzmann 
equation with a finite volume discretization in $v$, which preservs conservation laws, Frezzotti [12], see also the references therein, extended and compared variants of this method, in the context of the evaporation of binary mixtures which arise in metallurgy processes for instance. Notice that in these papers a Monte-Carlo method is still used to calculate the eight-fold integral arising in Boltzmann equation. Further, a fully deterministic finite volume in $v$, finite elements in $x$ method has been proposed recently by Rogier and Schneider [24] (and the references therein). Since it is directly deduced from an approximation of the Boltzmann kernel, this method preservs naturally the conservation laws, and Boltzmann's H-theorem at a semi-discrete level. Also, purely deterministic particle methods are presented in Niclot and Degond [19], Russo [25]. Another class of schemes, based on the theory of branching random processes, an exact algorithm for solving the Boltzmann equation is proposed in Ermakov and al. [11], Chauvun [7]. However this method has not been tested intensively and is costly.

This paper is organized as follows. In the first section, we introduce the main notations. In section II, we present the theoretical bases of the original DSMC method and of an improvment : the majorant frequence collision method. The last section is devoted to a direct derivation of a random particle method from the Boltzmann equation. In $[2,13,18]$, this method, combined with low discrepancy random choices, is called Finite Poinset Method.

\section{The Boltzmann equation}

The Boltzmann equation describes the evolution of a continuum of particles by mean of three variables : the time $t$, the position $x$ and the velocity $v$ of particles. In this model, the density of particles follows the equation

$$
\frac{\partial f}{\partial t}+v \cdot \frac{\partial f}{\partial x}=Q(f)=\int_{\mathbb{R}^{3} \times S^{2}}\left[f^{\prime} f_{*}^{\prime}-f f_{*}\right] B d v \cdot d \sigma,
$$

where $f=f(t, x, v), f_{*}=f\left(t, x, v_{*}\right), f^{\prime}=f\left(t, x, v^{\prime}\right)$ and $f_{*}^{\prime}=f\left(t, x, v_{*}^{\prime}\right)$. Given $\left(v, v_{*}, \sigma\right)$ in (1), we obtain the post-collisional velocities

$$
\begin{gathered}
v^{\prime}=\frac{v+v_{*}}{2}+\frac{|g|}{2} \sigma, v_{*}^{\prime}=\frac{v+v_{*}}{2}-\frac{|g|}{2} \sigma, \\
g=v-v_{*}, \\
B:=B(|g|,|g . \sigma|), B(0, a)=0 .
\end{gathered}
$$

Boltzmann's collision operator $Q(f)$ has the fundamental properties that it conservs mass, momentum, energy which means

$$
\int Q(f)(v)\left(1, v,|v|^{2}\right) d v=(0,0,0) .
$$


This follows from a more general symmetry identity ; for any function $\varphi$

$$
\begin{aligned}
\int Q(f)(v) \varphi(v) d v & =\frac{1}{4} \int Q(f)\left[\varphi+\varphi_{*}-\varphi^{\prime}-\varphi_{*}^{\prime}\right] d v \\
& =\frac{1}{2} \int Q(f)\left[\varphi+\varphi_{*}\right] d v .
\end{aligned}
$$

The deviation of (6) uses the particular form of $B$ in (4). Finally, a consequence of (6) is Boltzmann's H-theorem

$$
\int Q(f)(v) \ln f(v) d v \leq 0 .
$$

We refer to Cercignani [6], Truesdell and Muncaster [27] for a complete proof of these results. Notice that we have used in (2) a parametrization of the sphere which is adapted to the numerical methods. A more frequent presentation is to use a vector $w$ of the sphere, related to (1) by

$$
\begin{aligned}
g^{\prime}:=v^{\prime}-v_{*}^{\prime} & =|g| \sigma=g-2(g, w) w, \\
d \sigma & =4 \frac{|(g, w)|}{|g|} d w .
\end{aligned}
$$

This reduces the classical Very Hard Spheres model used for hypersonic flows in the upperatmosphere to

$$
B(|g|,|g . \sigma|)=K|g|^{\alpha}, 0 \leq \alpha<1 .
$$

Nowadays, the methods based on splitting the physical processes are widely used. A free transport is evaluated first, for a time interval $\Delta t_{f}$. Next, a spatially homogeneous collision step is performed, which is

$$
\frac{\partial f}{\partial f}=Q(f), 0 \leq t \leq \Delta t_{f} .
$$

Recently Desvillettes and Mischler [29] have proved that this splitting converge, as $\Delta t_{f}$ tends to zero, to the Di Perna-Lions [10] solution of Boltzmann equation.

Next, particle approximations of kinetic equations are based on the approximation of the density $f$ by a sum of Dirac masses

$$
f(t, x, v) \simeq \frac{1}{n_{\infty}} \sum_{i=1}^{N} \delta\left(x-x_{i}(t)\right) \delta\left(v-v_{i}(t)\right) .
$$

The positions $x_{i}(t)$ change during the free flow only, the velocities $v_{i}(t)$ change during the collision step only. $N$ denotes the total number of particles, $n_{\infty}$ is a reference number. Covering the computational domain with cells $C_{j}$ (say of uniform volume to simplify), this allows to define a local density $\rho_{j}$ in the cell $C_{j}$ by

$$
\rho_{j}(t)=n_{j}(t) / n_{\infty}
$$


where $n_{j}$ is the number of $x_{i}(t)$ which belong to $C_{j}$. Sampling a maxwellian. distribution, for instance, is now possible.

Using particles, the main difficulty in the numerical resolution of (1) is the collision step. From (9), a local distribution in the cells $C_{j}$ is deduced, still denoted $f$,

$$
f(t, v)=\frac{1}{n_{\infty}} \sum_{i=1}^{n} \delta\left(v-v_{i}(t)\right), \rho=\frac{n}{n_{\infty}}
$$

where $n$ stands for $n_{j}$ and new indices for $v$ have been introduced compared to (9). And the question is to solve (8) with a distribution like (10).

\section{Direct Simulation Monte-Carlo}

Although the numerical techniques for simulating (8) are often obtained using heuristic arguments based on physical ideas, it seems possible to relate a large class of methods to the master equation, associated with (8). This class contains Bird's original algorithm [5], and variants are compared in Koura [15]. A very good description of these variants can be found in Ivanov and Rogasinsky [14] as well as relations with the master equation, and we will follow his presentation in this section. But these relations are frequently quoted : Nanbu [17], Belotserkovsky, Erofeyev and Yanitsky [4]. Also this approach gave rise to mathematical convergence proofs for small initial data (Wagner [28]) or for delocalized collisions (Pulvirenti, Wagner and Zavelani-Rossi [23]), or for simplified models up to the fluid limit (Perthame and Pulvirenti [21]).

The homogeneous master equation for the $n$-particle distribution function has the form (see Cercignani [6])

$$
\begin{gathered}
\frac{\partial}{\partial t} h_{n}(t, V)=\frac{\rho}{n} \sum_{1 \leq i<j \leq n} \int_{S^{2}}\left[h_{n}\left(t, V_{i j}^{\prime}\right)-h_{n}(t, V)\right], B_{i j}(\sigma) d \sigma \\
B_{i j}(\sigma):=B\left(\left|g_{i j}\right|,\left|g_{i j} . \sigma\right|\right), g_{i j}=v_{i}-v_{j}
\end{gathered}
$$

where $\rho$ is the local density in the cell (see (10)), $V$ and $V_{i j}^{\prime}$ are $3 n$-dimensional vectors, $V=\left(v_{1}, \ldots, v_{n}\right), V_{i j}^{\prime}=\left(v_{1}, \ldots, v_{i}^{\prime}, \ldots, v_{j}^{\prime}, \ldots, v_{n}\right)$, and $\left(v_{i}^{\prime}, v_{j}^{\prime}\right)$ are the post-collisional velocities obtained colliding $\left(v_{i}, v_{j}, \sigma\right)$ according to $(2)$ with $g_{i j}=v_{i}-v_{j}$. The relation between (9) and (11) is as follows. Integrating (11) against $d v_{2} \ldots d v_{n}$ and using the symmetry of $h$ in $\left(v_{1}, \ldots, v_{N}\right)$ when the initial data is symmetric, we obtain

$$
\begin{gathered}
\frac{\partial}{\partial t} h_{n}^{(1)}\left(t, v_{1}\right)=\rho \frac{n-1}{n} \int_{S^{2}}\left[h_{n}^{(2)}\left(t, v_{1}^{\prime}, v_{2}^{\prime}\right)-h_{n}^{(2)}\left(t, v_{1}, v_{2}\right)\right] B_{12}(\sigma) d \sigma d v_{2}, \\
h_{n}^{(j)}\left(t, v_{1}, \ldots v_{j}\right)=\int h_{n}(t, V) d v_{j+1} \ldots d v_{n} . \text { Now, we take the initial date } \\
h_{n}(0, V)=\prod_{i=1} f\left(0, v_{i}\right) / \rho,
\end{gathered}
$$


and we expect from the propagation of chaos that $h_{n}$ factorizes as $N$ tends to infinity, which means that

$$
h_{n}^{(2)}\left(t, v_{1}, v_{2}\right) \simeq h_{n}^{(1)}\left(t, v_{1}\right) h_{n}^{(1)}\left(t, v_{2}\right)
$$

Then, (13) exactly yields (8) with $f=\rho h=l i m \rho h_{n}^{(1)}$ as $N$ tends to infinity.

The DSMC method aims to solve the equation (11), which is linear, rather than the nonlinear equation (8). This assumes that we have a large number of particles in each cell, but all methods do so. Also, due to the linearity of (11), its solution is represented by a Markov process, which leads the numerical procedure.

Let us describe one algorithm, details, variants, improvments can be found in $[5,14,15,17,4]$.

The collision frequency is defined as

$$
\begin{gathered}
\nu(V)=\frac{\rho}{n} \sum_{i<j} B_{T, i j} \\
B_{T, i j}=\int_{S^{2}} B_{i j}(\sigma) d \sigma .
\end{gathered}
$$

Then, (11) can be written also

$$
\partial_{t} h_{n}(t, V)+\nu(V) h_{n}(t, V)=\frac{\rho}{n} \sum_{i<j} \int_{S^{2}} h_{n}\left(t, V_{i j}^{\prime}\right) B_{i j}(\sigma) d \sigma
$$

The probabilistic representation of the solution to (11) is

$$
h_{n}(t, V)=E\left[h_{n}(0, V(t))\right]
$$

where the expectation is taken according to a probability space on which the jump process $V(t)$ is built with generator in (11) (see Iketa and Watanabe [13]).

The DSMC method is just to sample this Markov process. Its initial state $V_{o}$ is assumed to be known or sampled according to the initial distribution (14) at time $t_{o}=0$. Then, the DSMC method generates a sequence of random times $t_{1}, t_{2}, \ldots, t_{k}, \ldots$ and at each time $t_{k}$ a new 3 -dimensional state $V_{k}$ is computed, until we reach $t_{k}=\Delta t_{f}$ the final time. From $\left(t_{k}, V_{k}\right)$ we compute $\left(t_{k+1}, V_{k+1}\right)$ as follows :

First step : The times $t_{k}$ are sampled according to a Poisson law of parameter $\nu\left(V_{k}\right)$ i.e.

$$
t_{k+1}-t_{k}=-\nu^{-1}\left(V_{k}\right) \ln (\mathrm{rand})
$$

where rand is random number uniformly distributed on the interval $(0,1)$. 
Second step : A collisional pair $\left(v_{i}^{o}, v_{j}^{o}\right)$ with $i<j$ and a collision parameter $\sigma \in S^{2}$ are sampled with the probability

$$
p(i, j, \sigma)=\frac{B_{i j}(\sigma)}{\nu(V)}
$$

Once $(i, j, \sigma)$ are known (from the current value $V_{k}$ of $V$ ) we just choose $V_{k+1}=$ $V_{i j}^{\prime}$, using the rule (2), (12).

In practice, the second step is performed so as to decouple the choice of the pair $(i, j)$ and the choice of $\sigma \cdot(i, j)$ is sampled with the probability

$$
q(i, j)=\frac{B_{T, i j}}{\nu(V)}
$$

using this pair, $\sigma$ is sampled with the probability

$$
q^{\prime}(\sigma)=\frac{B_{i j}(\sigma)}{B_{T, i j}}
$$

This method, usually called "time counter", is rather expensive (of order $n$ per time step using a fast algorithm to sample (21)). A classical cost reduction to 1 per time step can be achieved using the "majorant collision frequence" technique where an upper bound $\lambda$ of $B_{T}$ is supposed to be known

$$
B_{T, i j} \leq \lambda, \text { for all } i, j
$$

Then, (11) is rewritten again as

$$
\partial_{t} h_{n}+\rho(n-1) \lambda h_{n}=\frac{\rho}{n} \sum_{i<j}\left[\left(\lambda-B_{T, i j}\right) h_{n}+\int_{S^{2}} h_{n}\left(V_{i j}^{\prime}\right) B_{i j}(\sigma) d \sigma\right]
$$

The Markov process consists now in

First step : $t_{k+1}-t_{k}=-[\rho(n-1) \lambda]^{-1} \ln ($ rand $)$.

Second step : A pair $\left(v_{i}, v_{j}\right)$ is uniformly chosen in $V_{k}$, and a new random number, rand' too. If

$$
\lambda \operatorname{rand}^{\prime} \geq B_{T, i j}
$$

the collision is "fictitious" and we keep $V_{k+1}=V_{k}$ (no collision occurs). In the other case, we can perform the

Third step : $\left(v_{i}, v_{j}\right)$ is chosen as the collisional pair and $\sigma$ is sampled following (21). The new $V_{k+1}$ is computed as before using (2).

The "majorant collision frequence" reduces the computational cost to the order 
1 per time step-compare to the original "time counter". On the other hand, it is nothing but a rejection method and thus its variance is worse in particular when $\lambda$ is too large.

Further variants are possible, introducing fixed time steps for instance. This makes it possible to relate these methods directly to the Boltzmann equation (1) rather than to the master equation.

\section{Random particle methods}

The first algorithm which relies directly on the Boltzmann equation seems to be due to Nanbu [16]. A first theoretical investigation of this method can be found in Babovski [2] and Babovski-Ilner [3]. Further research in this direction led Illner and Neunzert [30], Neunzert, Gropengiesser and Struckmeier [18] to introduce the Finite Pointset Method where low discrepancy methods are used to sample the random variables.

Here, we just derive a simulation scheme directly from (8), (10) and we send the reader to the above references for practical details and further variants.

First, a smaller timestep $\Delta t$ is chosen to reach $\Delta t_{f}$, the timestep for free flow. The homogeneous Boltzmann equation is approximated by Euler scheme

$$
f^{k+1}(v)=f^{k}(v)+\Delta t Q\left(f^{k}\right)(v)
$$

where $f^{k}(v)$ is an approximation of $f(k \Delta t, v)$. We assume that $f^{k}$ is given by a sum of Dirac masses

$$
f^{k}(v)=\frac{1}{n_{\infty}} \sum_{i=1}^{n} \delta\left(v-v_{i}\right), v_{i}:=v_{i}^{k},
$$

and the method approximates $f^{k+1}$ in (20) by another combination of exactly $n$ new Dirac masses at the points $v_{i}^{k+1}$. To do so, we first notice that $Q$ acts on measures. Indeed, for any continuous test function $\varphi,(25)$ is equivalent, using (6) to

$$
\begin{aligned}
& \int f^{k+1}(v) \varphi(v) d v=\int f^{k}(v) \varphi(v) d v \\
- & \frac{\Delta t}{2} \int f^{k} f_{*}^{k}\left(\varphi+\varphi_{*}\right) B_{T}(|g|) d v d v_{*} \\
+ & \left.\frac{\Delta t}{2} \int f^{k} f_{*}^{k}\left(\varphi^{\prime}+\varphi_{*}^{\prime}\right) B(|g|),|g \cdot \sigma|\right) d v d v_{*} d \sigma .
\end{aligned}
$$

Inserting the distribution (26) in this relation, we obtain using the notation (12), (17) and after straightforward calculations

$$
\int f^{k+1}(v) \varphi(v) d v=
$$




$$
\begin{aligned}
& \frac{1}{2 n_{\infty}(n-1)} \sum_{\substack{i, j=1 \\
i \neq j}}^{n}\left\{\left(\varphi\left(v_{i}\right)+\varphi\left(v_{j}\right)\right)\left(1-\rho \Delta t B_{T, i j}\right)\right. \\
+ & \left.\int_{S^{2}} \rho \Delta t B_{i j}(\sigma)\left(\varphi\left(v_{i}^{\prime}\right)+\varphi\left(v_{j}^{\prime}\right)\right) d \sigma\right\},
\end{aligned}
$$

where $\underline{\rho}=(n-1) / n_{\infty}$ and $\left(v_{i}^{\prime}, v_{j}^{\prime}\right)$ is obtained as a collision of $\left(v_{i}, v_{j}\right)$ with the parameter $\sigma$ as in (2). Notice that, due to the assumption (4) the sum in (28) is indeed on $i \neq j$.

Next, and following the above reference, we assume that the number of particles is even

$$
n=2 p,
$$

and we denote by $P$ the set of all possible pairings of $E=\left\{v_{1}, \ldots v_{n}\right\}$ i.e. $P$ is the set of all possible covering of $E$ by $p$ pairs $\left\{\left(v_{1}, w_{1}\right),\left(v_{2}, w_{2}\right), \ldots,\left(v_{p}, w_{p}\right)\right\}$. Now for any function $q$, we have

$$
\frac{1}{2(n-1)} \sum_{i \neq j} \varphi\left(v_{i}, v_{j}\right)=\frac{1}{|P|} \sum_{P} \sum_{i=1}^{p} \varphi\left(v_{i}, w_{i}\right)
$$

where $|P|$ is the number of all possible pairings of $E$. Therefore, (28) can also be written

$$
\begin{gathered}
\int f^{k+1}(v) \varphi(v) d v= \\
\frac{1}{n_{\infty}} \frac{1}{|P|} \sum_{P} \int_{0}^{1}\left\{\sum_{i=1}^{p} \chi\left(\underline{\rho} \Delta t B_{T, i} \leq s \leq 1\right)\left(\varphi\left(v_{i}\right)+\varphi\left(w_{i}\right)\right)\right. \\
\left.+\quad \chi\left(0 \leq s \leq \rho \Delta t B_{T, i}\right) \int_{S^{2}}\left(\varphi\left(v_{i}^{\prime}\right)+\varphi\left(w_{i}^{\prime}\right)\right) d \mu_{i}(\sigma)\right\} d s
\end{gathered}
$$

where $\left(v_{i}^{\prime}, w_{i}^{\prime}\right)$ is obtained as a collision of $\left(v_{i}, w_{i}\right)$ with the parameter $\sigma, \chi$ is the indicator function and

$$
\begin{gathered}
B_{i}(\sigma)=B\left(\left|v_{i}-w_{i}\right|,\left|\left(v_{i}-w_{i}\right) \cdot \sigma\right|\right), B_{T, i}=\int_{S^{2}} B_{i}(\sigma) d \sigma \\
d \mu_{i}\left(\sigma=\frac{B_{i}(\sigma)}{B_{T, i}} d \sigma .\right.
\end{gathered}
$$

At this level a restriction on the time, step has to be imposed

$$
\underline{\rho} \Delta t \max _{1 \leq i \leq p} B_{T, i} \leq 1
$$

Finally, a Monte-Carlo procedure is used to evaluate integral in (31) against the probability measures

$$
\frac{1}{|P|} \sum_{P} \int_{0}^{1}(\ldots) d s, \text { and } \int_{S^{2}}(\ldots) d \mu_{i}(\sigma) .
$$


This means that a special pairing $\left(v_{1}, w_{1}\right), \ldots,\left(v_{p}, w_{p}\right)$ is chosen with an uniform law in $P$, a random number $s \in(0,1)$ is chosen with a uniform law as well and a unit vector $\sigma_{i}$, for each $i$, is finally chosen with the probability $d \mu_{i}(\sigma)$. This replaces (31) by a random approximation

$$
\begin{gathered}
\int f^{k+1}(v) \varphi(v) d v= \\
\frac{1}{n_{\infty}} \sum_{i=1}^{p}\left\{\chi\left(\underline{\rho} \Delta t B_{T, i} \leq s \leq 1\right)\left(\varphi\left(v_{i}\right)+\varphi\left(w_{i}\right)\right)\right. \\
\left.+\chi\left(0 \leq s \leq \underline{\rho} \Delta t B_{T, i}\right)\left(\varphi\left(v_{i}^{\prime}\right)+\varphi\left(w_{i}^{\prime}\right)\right)\right\},
\end{gathered}
$$

which completely identifies $f^{k+1}$ as a sum of $n=2 p$ Dirac masses,

$$
f^{k+1}(v)=\frac{1}{n_{\infty}} \sum_{i=1}^{p}\left[\delta\left(v-v_{i}^{k+1}\right)+\delta\left(v-w_{i}^{k+1}\right)\right] .
$$

when $\rho \Delta t B_{T, i} \leq s$ then $\left(v_{i}^{k+1}, w_{i}^{k+1}\right)=\left(v_{i}^{k}, w_{i}^{k}\right)$, no collision is performed. When $s \leq \rho \Delta t B_{T, i}$ then $\left(v_{i}^{k+1}, w_{i}^{k+1}\right)=\left(v_{i}^{\prime}, w_{i}^{\prime}\right)$, the collision is performed between $\left(v_{i}, w_{i}\right)$ with the parameter $\sigma_{i}$.

Notice that when the number of particles $n$ is odd, a systematic error is done. It can be corrected by choosing $s$ uniformly on $\left(0, \frac{n-1}{n}\right)$ rather than $(0,1)$ for $n$ odd.

Also, variants are possible : one can choose $s_{i}$ for each $i$ rather than a global $s$; this is equivalent to inverting $\int_{0}^{1}$ and $\sum_{i}$ in (31). Another variant consists in using a majorant collision frequency as described in section II ; the advantage is to use the same number of cycles for each cell which is better for vectorization. In order to avoid the restriction (34) on the timestep, L. Desvillettes [9] proposed also a variant where $\Delta t$ can be as large as necessary. His method is also very close to the DSMC method and works pretty well on practical examples.

Finally, we would like to point out that the DSMC method and this random particle method are very similar. In some sense the DSMC method uses a time step $n$ time smaller and performs one collision per timestep. Here the timestep is $n$ time larger and $n$ possible collisions are done at once so that the computational time between the majorant frequence technique and the present method are comparable. Comparisons can be found in Struckmeier and Steiner [26]. Recall that the Monte-Carlo method is now reduced to the generation of independant variables with a specified repartition law. This is a whole problem in itself.

\section{References}

[1] V.V. Aristov and F.G. Cheremisin. Conservative splitting method for 
solving the Boltzmann equation. USRR Comput. Math. Math. Phys. 20 (1980), p. 191-207 (in russian).

[2] H. Babovsky. On a simulation scheme for the Boltzmann equation. Math. Meth. in the Appli. Sc. 8, (1986) p. 223-233.

[3] H. Babovsky and R. Illner. A convergence proof for Naubu's simulation method for the full Boltzmann equation, SIAM J. Numer. Anal. 26, 1 (1989), p. 45-65.

[4] O.M. Belotserkovsky, A.I. Erofeyev and V.E. Yanitsky. Direct simulation of problems in aerohydrodynamics in Numerical Methods in Fluid Dynamics. N.N. Yanenko and Y.I. Shokin Editors. MIR, Moscow (1986).

[5] G.A. Bird. Molecular gas dynamics, Clarendon Press, Oxford (1976).

[6] C. Cercignani The Boltzmann Equation and its applications. Springer, Berlin (1988).

[7] B. Chauvin. Branching processes, trees and the Boltzmann equation. To appear in Proceedings du congrès Probabilités Numériques INRIA 1993. E. Pardoux and D. Tallay Editors.

[8] F. Coron and B. Perthame. Numerical passage from kinetic to fluid equations. SIAM J. Num. Anal. 28(1), (1991), p. 26-42.

[9] L. Desvillettes. A vectorizable simulation method for the Boltzmann equation. To appear in Meth. Math. Num. Anal.

[10] R.J. Di Perna and P.L. Lions. On the Cauchy problem for Boltzmann equations : global existence and weak stability. Ann. Math. 130 (1989) p. $321-366$.

[11] S.M. Ermakov, V.V. Nekrutkin, A. Ya Proshkin and A.F. Sizova, On Monte-Carlo solution of nonlinear kinetic equations. Dokl. Akad. Nauk. SSSR 230(2) (1976) p. 261-263 (in russian).

[12] A. Frezzotti. Numerical study of the strong evaporation of a binary mixture. Fluid Dyn. Research 8 (1991), p. 175-187.

[13] N. Ikeda and S. Watanabe. Stochastic differential equations and diffusion processes. North-Holland, Amsterdam (1981).

[14] M.S. Ivanov and S.V. Rogasinsky. Analysis of numerical techniques of the direct simulation Monte-Carlo method in the rarefied gas dynamics. Sov. J. Numer. Anal. Math. Modelling 3(6), (1988), p. 453-465. 
[15] K. Koura. A sensitive test for accuracy in evaluation of molecular collision number in the direct simulation Monte-Carlo method. Phys. Fluid A2(7) (1990), p. 1287-1289.

[16] K. Nanbu. Direct simulation scheme derived from the Boltzmann equation. I. Monocomponent gases. J. Phys. Soc. Japan 49 (1980) p. 2042-2049.

[17] K. Nanbu. Stochastic solution method of the Master equation and the model Boltzmann equation. J. Phys. Soc. Japan 52 (1983) p. 2654-2658.

[18] H. Neunzert, F. Gropengiesser and J. Struckmeier. Computational methods for the Boltzmann equation in : Applied and Industrial Mathematics (Ed. R. Spigler), Kluwer Acad. Publ. Dordrecht (1991) p. 111-140.

[19] B. Niclot, P. Degond. Numerical analysis of the weighted particle method applied to the semiconductor Boltzmann equation. Numer. Math. 55 (5) (1989), p. 599-618.

[20] B. Perthame, Boltzmann type schemes for gas dynamics and the entropy property. SIAM J. Num. Anal. 27 (6) (1990), p. 1405-1421.

[21] B. Perthame and M. Pulvirenti. On a system of particles approximating scalar conservation laws. To appear in Asymp. Anal.

[22] D.I. Pullin. Direct simulation methods for compressible inviscid ideal-gas flow. J. Comput. Phys. 34 (1980), p. 231)244.

[23] M. Pulvirenti, W. Wagner and M.B. Zavelani Rossi. Convergence of particle schemes for the Boltzmann equation. Prepring no 49, Institut fur Angewandte Analysis und Stochastik, Berlin 1993.

[24] F. Rogier and J. Schneider. A direct method for solving the Boltzmann equation. To appear in Tr. Th. and Stat. Phys:

[25] G. Russo, A particle method for collisional kinetic equations. I. Basic theory and one-dimensional results. J. Comput. Phys. 87 (1990) p. 270300 , and Part II to appear in J. Comput. Phys.

[26] J. Struckmeier and K. Steiner. A comparison of simulation methods for rarefied gas flows. Preprint 91, Arbeitsgruppe technomathematik, Univ. Kaiserslautern (1993).

[27] C. Truesdell and R.G. Muncaster. Fundamentals of Maxwell's kinetic theory of a simple monoatomic gas. Academic Press, New York (1980). 
[28] W. Wagner. A convergence proof of Bird's direct simulation Monte-Carlo method for the Boltzmann equation. J. Stat. Phys. 66, 314 (1992) p. 10111044.

[29] L. Desvillettes and S. Mischeler. Work in preparation.

[30] R. Illner and H. Neunzert. On simulation methods for the Boltzmann Equation. Transp. Th. and Stat. Phy. 16, 2 and 3 (1987), p. 141-154. 


\section{EDITEUR}

INRIA - Domaine de Voluceau - Rocquencourt - B.P. 105 - 78153 LE CHESNAY Cedex (France) 